\title{
Evoluindo um processo de elicitação de requisitos com foco na seleção da técnica mais adequada de elicitação
}

\author{
Glívia Barbosa ${ }^{1}$, Marcelo Werneck ${ }^{1}$, Ismael Silva ${ }^{1,2}$, Ulisses Fernandes ${ }^{1}$ \\ ${ }^{1}$ Instituto de Informática - Pontifícia Universidade Católica de Minas Gerais (PUC \\ Minas) - Belo Horizonte - MG - Brasil \\ ${ }^{2}$ bes - Belo Horizonte - MG - Brasil \\ \{mwerneck\}@pucminas.br, \{gliviaangelica,ufernandes\}@gmail.com, \\ \{ismael\}@besconsulting.com.br
}

\begin{abstract}
Resumo. Este trabalho apresenta um processo focado na seleção da técnica de elicitação de requisitos. Na tentativa de garantir que os requisitos relevantes para o sistema sejam elicitados de forma correta e completa, um conjunto de técnicas de elicitação pode ser aplicado com o intuito de auxiliar os analistas e usuários na identificação e definição desses requisitos. Porém, a elicitação não se resume apenas à aplicação de uma técnica, mas também à colaboração entre analistas e clientes. Este trabalho apresenta a evolução de um processo de elicitação de requisitos com foco na seleção da técnica de elicitação. A seleção é realizada de acordo com as características do projeto em questão e seus envolvidos. Novos experimentos mostraram que as elicitações realizadas contemplaram requisitos que correspondem às necessidades do cliente, de acordo com verificações realizadas pelo mesmo.
\end{abstract}

\begin{abstract}
This work presents a process focused on the selection of the requirements elicitation technique. In an attempt to ensure that the relevant system requirements are correctly and completely elicited, a set of techniques can be applied aiming at helping the analysts and users identify and define these requirements. However, the elicitation is not simply the application of a technique but also the cooperation between analysts and clients. This work presents the evolution of the requirements elicitation process focused on technique selection. The selection is performed according to characteristics of the project and its stakeholders. New experiments have shown that the elicitations comprised requirements that correspond to the clients' needs according to verifications carried out by themselves.
\end{abstract}

\section{Introdução}

A Engenharia de Requisitos contempla um conjunto de atividades relacionadas à definição e gerência dos requisitos. Dentre essas atividades, podemos destacar: elicitar, analisar, documentar, validar estes requisitos e, durante todo o desenvolvimento de software, gerenciá-los [Pfleeger, 2004].

Uma etapa crítica da engenharia de requisitos é a elicitação, fase complexa da definição de requisitos e de todo processo de desenvolvimento de software, uma vez que é base para todas as etapas posteriores [Belgamo e Martins, 2000]. Elicitar requisitos requer um processo iterativo, que deve acontecer de forma colaborativa envolvendo analistas, usuários e aplicação de técnicas [Freitas, Borges e Araújo, 2007]. 
Um estudo realizado pelo Standish Group [Standish report, 1995], com 350 companhias e 8.000 projetos indica que 16,2\% dos projetos são concluídos com sucesso; $52,7 \%$ são considerados problemáticos, pois não atendem às necessidades dos usuários com custos excessivos e $31,1 \%$ são cancelados antes de serem completados. Buscando identificar quais fatores levam à distribuição apresentada, nova pesquisa foi realizada e 8 fatores foram identificados como críticos, dos quais 3 estão relacionados à elicitação e gerência dos requisitos. São eles: "Requisitos incompletos", "Falta de envolvimento do usuário" e "Mudanças de requisitos e especificações".

A forma como a elicitação de requisitos é feita influencia o produto desenvolvido. Os requisitos levantados são um indicador importante para o fracasso ou sucesso do projeto de um software. Requisitos mal levantados ou mal interpretados geram retrabalho, custos e prazos extras, além de insatisfação do cliente.

Existem técnicas que buscam auxiliar os analistas e os usuários na identificação dos requisitos do sistema, contudo, a complexidade dessa etapa de elicitação se deve não apenas a fatores técnicos, mas também humanos. [Barbosa et al., 2009]

As técnicas de elicitação auxiliam o desenvolvimento de software, mas os problemas essenciais da elicitação são um desafio que ainda precisa ser superado. Uma possível solução mesclada às técnicas e ferramentas que poderá resolver estes problemas essenciais é o uso de um processo que promova um trabalho colaborativo entre a equipe e os usuários certos, ou seja, aqueles que realmente contribuem para a identificação dos requisitos [Freitas, Borges e Araújo, 2007].

Escolher e aplicar uma técnica de elicitação não é uma tarefa trivial, porém reduzir a fase de elicitação à utilização de técnicas apenas não garante que os requisitos identificados satisfaçam o cliente final [Belgamo e Martins, 2000].

Algumas técnicas que facilitam a comunicação entre clientes e analistas têm sido usadas visando minimizar problemas encontrados na elicitação de requisitos. Tem sido observado, porém, que a forma com que estas técnicas têm sido aplicadas ainda não solucionou os problemas da elicitação de requisitos. Constata-se que, em muitos casos, os analistas insistem em utilizar somente a técnica sem uma preparação anterior e em outras situações a mesma técnica é utilizada para elicitar requisitos de todos projetos de uma empresa [Hickey e Davis, 2002]. Além disso, pouca pesquisa tem sido focada na identificação das técnicas de elicitação mais adequadas [Dieste, Lopez e Ramos, 2008].

Considerando a elicitação de requisitos uma fase crítica no desenvolvimento de software, inicialmente propusemos um processo de elicitação de requisitos com foco na escolha da técnica mais adequada para elicitação de acordo com as características do domínio do problema e escopo do projeto. A utilização deste processo inicial revelou vários benefícios através dos experimentos realizados, como, uma maior interação entre analistas e usuários e o auxilio significativo na seleção da técnica a ser utilizada na identificação dos requisitos. Tais benefícios resultaram no aumento da qualidade dos requisitos elicitados e mostraram que esta foi uma solução que contribuiu para que os requisitos levantados correspondessem às reais necessidades dos usuários.

Contudo, a seleção de uma técnica para realizar o levantamento de requisitos de um software exige uma investigação mais aprofundada. $\mathrm{O}$ mecanismo proposto, nesta primeira versão do processo, descrito em [Barbosa et al., 2009] para apoiar o Analista de Requisitos na seleção da técnica de elicitação foi confrontado com estudos recentes 
neste domínio. Foi observado que mais pesquisas são necessárias, mesmo considerando que um criterioso estudo, baseado na literatura relacionada, tenha sido recentemente realizado [Carrizo, Dieste e Juristo, 2008] e [Dieste, Lopez e Ramos, 2008].

Após analisados os resultados dos experimentos iniciais, realizados com a primeira versão proposta desse processo, bem como os experimentos que permitiram confrontar os resultados dos experimentos inicias e os trabalhos relacionados citados, concluímos que para oferecer maior apoio na seleção da técnica de elicitação no levantamento de requisitos é importante considerar características do(s) usuário(s), analista(s) de requisitos, escopo e do domínio/contexto do projeto.

Neste trabalho, propomos uma evolução do processo para elicitação de requisitos com foco na seleção da técnica. Nesta nova versão, foram realizadas atualizações em algumas atividades propostas inicialmente e o processo de elicitação, atualizado, foca na escolha da técnica de elicitação com base nas características do projeto, no perfil do analista e do(s) usuário(s) e na colaboração entre envolvidos. Assim, mais características das técnicas e dos projetos são levadas em consideração.

Este trabalho está organizado da seguinte maneira: a Seção 2 descreve os conceitos relacionados à Engenharia de Requisitos e Técnicas de Elicitação. A Seção 3 apresenta os trabalhos relacionados. A evolução do processo proposto e os resultados obtidos são descritos na Seção 4 enquanto a Seção 5 conclui o trabalho.

\section{Elicitação de Requisitos}

A elicitação corresponde ao processo de levantamento de requisitos. Nesta fase, são levantados os objetivos principais do sistema, identificando assim os problemas apresentados pelos usuários e/ou clientes. Em um segundo momento, são identificadas as possíveis soluções para os problemas e objetivos detectados [Lauesen, 2002].

Durante o processo de elicitação, é necessário manter um contato com os usuários e clientes do sistema para entender suas reais necessidades obtendo assim os requisitos do software [Pfleeger, 2004]. Os problemas de elicitação de requisitos não podem ser solucionados apenas com uma abordagem tecnológica, uma vez que o conjunto formado por aspectos sociais, pessoas, informações e contextos assumem importância significativa na elicitação [Belgamo e Martins, 2000].

$\mathrm{Na}$ tentativa de minimizar os problemas encontrados na atividade de elicitação de requisitos, existem algumas técnicas que facilitam a comunicação entre clientes e analistas. Estas técnicas são conhecidas como técnicas de elicitação de requisitos.

\subsection{Técnicas de Elicitação de Requisitos}

As técnicas de elicitação surgiram para auxiliar na identificação dos requisitos junto aos usuários. Uma técnica deve explorar características específicas do problema sendo tratado no desenvolvimento de um sistema. Como as características dos problemas variam, é necessário um repertório de métodos para cada classe de problemas [Belgamo e Martins, 2000]. Há muitas técnicas de elicitação publicadas na literatura [Dieste, Lopez e Ramos, 2008]. Entretanto, informações são ainda capturadas, em muitos casos, utilizando somente entrevistas, apesar de haver clara evidência de que entrevistas tradicionais nem sempre são a melhor forma de se extrair requisitos [Carrizo, Dieste e Juristo, 2008]. Nesta seção, apresentamos brevemente algumas técnicas de elicitação. 
As Oficinas de Requisitos são reuniões com participação dos desenvolvedores, usuários e outros interessados para definição de requisitos de um sistema em conjunto. A técnica JAD (Joint Application Development) é um exemplo de oficina. JAD visa reunir autoridades representativas e gerenciais em um workshop organizado para promover decisões [Belgamo e Martins, 2000]. Sua aplicação é recomendada quando a necessidade de consenso entre os usuários do sistema se torna fator importante para o desenvolvimento do software. O objetivo dessa técnica é garantir que os usuários se mantenham comprometidos com o levantamento dos requisitos do sistema.

A Prototipagem é uma técnica utilizada com frequiência na elicitação de requisitos. Existem dois tipos de protótipos: os descartáveis e os evolutivos. Protótipos descartáveis são criados com a função de ilustrar para os usuários e/ou clientes do sistema o que o analista entendeu sobre os requisitos que deverão ser contemplados no produto. Essa prototipagem deve ser feita rapidamente e ser concluída em no máximo alguns dias. Protótipos evolutivos são reaproveitados durante a construção do sistema.

A Entrevista é uma técnica que ajuda na captura de conhecimento sobre o domínio do problema, porém, para identificar a solução desses problemas, muitas vezes, se torna necessário utilizar outras técnicas. [Lauesen, 2002]

O uso de Questionários é muito utilizado quando os analistas identificam a necessidade de coletar informações de muitos usuários ao mesmo tempo [Lauesen, 2002]. Quando aplicado, cada usuário responde o questionário individualmente e posteriormente os requisitos são identificados através de análise de respostas fornecidas.

Em sessões de Brainstorming, um grupo de pessoas é reunido, um cenário simulado e um assunto discutido para elicitar os requisitos. As pessoas participantes devem se sentir confortáveis o bastante para discutir o assunto sem se sentirem intimidadas. Nenhuma idéia é descartada. Todas idéias são boas idéias [Lauesen, 2002].

\section{Trabalhos Relacionados}

Em [Belgamo e Martins, 2000], é realizada uma análise que visa estudar, comparar e apresentar diferenças e semelhanças entre algumas técnicas de elicitação selecionadas para enfrentar os problemas de elicitação de requisitos. Os autores propuseram um conjunto de parâmetros para avaliação e classificação de algumas técnicas estudadas.

Outro estudo relacionado ao tema foi realizado por [Batista e Carvalho, 2003]. Os autores apresentaram alguns parâmetros relacionados aos projetos e às técnicas que devem ser analisadas no momento da escolha da técnica de elicitação.

No trabalho proposto por [Freitas, Borges e Araújo, 2007], é descrito um processo colaborativo para elicitação de requisitos. Os autores apresentam um processo e uma ferramenta de apoio à colaboração e à negociação na fase de levantamento e definição dos requisitos do sistema. Por meio de um estudo de caso, os autores concluíram que a proposta contribuiu para o desempenho da atividade e aumentou as iniciativas de comunicação entre os stakeholders. Como resultado, a atividade de elicitação ganhou características mais ativas e participativas.

O artigo [Dieste, Lopez e Ramos, 2008] apresenta algumas recomendações sobre as situações em que cada técnica é útil. Estas recomendações são baseadas em uma revisão sistemática de vários estudos empíricos da literatura. 
O trabalho [Carrizo, Dieste e Juristo, 2008] propõe um framework para apoiar a tomada de decisão quanto à melhor técnica de elicitação de acordo com as características do projeto em questão. O framework define como cada técnica responde a distintas características de projetos.

Este trabalho se diferencia dos citados, pois definiu, inicialmente, um processo de elicitação de requisitos com suporte à seleção da técnica de elicitação de requisitos. Em seguida o mecanismo usado para apoiar na seleção da técnica, na primeira versão do processo baseado nos parâmetros propostos por [Belgamo e Martins, 2000] e [Batista e Carvalho, 2003], foi confrontado com recentes estudos apresentados por [Dieste, Lopez e Ramos, 2008] e [Carrizo, Dieste e Juristo, 2008]. Finalmente, a partir da análise apresentada em [Barbosa e Barbosa, 2009], foi possível realizar a evolução do processo de elicitação de requisitos com foco na integração entre os envolvidos e no suporte à seleção da técnica de elicitação de requisitos, conforme será apresentado a seguir.

\section{Evolução do Processo de Elicitação de Requisitos Proposto}

O processo de elicitação proposto envolve, em sua execução, os seguintes papéis: Gerente de Projeto, Analista de Requisitos, Cliente/Usuário. Nas próximas seções será demonstrado o processo em sua primeira versão, como foi identificada a necessidade de evoluí-lo e as atualizações realizadas no mesmo.

\subsection{Processo de Elicitação de Requisitos Proposto (Primeira Versão)}

O processo de elicitação de requisitos proposto em [Barbosa et al., 2009] é composto de 5 atividades, descritas a seguir:

A primeira atividade, "Identificar o Contexto do Projeto" visa contextualizar o Analista de Requisitos sobre o Projeto que será desenvolvido. O Analista obtém informações, através de uma reunião com o Gerente do Projeto, relacionadas ao escopo, premissas, restrições e informações relacionadas ao domínio do negócio do cliente antes de realizar um primeiro contato direto com o(s) usuário(s) do sistema. Ao final desta atividade, é elaborado um Glossário com os principais termos relevantes relacionados ao domínio de negócio do cliente, identificados pelo analista. Este Glossário poderá ser atualizado durante todo o processo de elicitação.

A segunda atividade, "Realizar Apresentação Inicial" visa conscientizar os envolvidos, através de uma reunião, sobre a importância da colaboração do(s) usuário(s) dentro do processo de elicitação de requisitos. Durante a reunião, o Analista de Requisitos também coleta informações sobre os papéis e responsabilidades dos envolvidos no processo de elicitação. Estas informações serão estruturadas em uma Matriz de Responsabilidades.

A terceira atividade, "Selecionar a Técnica de Elicitação de Requisitos" tem como objetivo auxiliar o Analista de Requisitos na escolha da técnica de elicitação, para realizar o levantamento de requisitos, mais adequada de acordo com as características do projeto e da organização.

O processo descrito não restringe o número de técnicas de elicitação que podem ser analisadas para seleção, entretanto, para realizar nossos experimentos, definimos que o processo seria baseado em um universo de 5 técnicas. Procuramos escolher técnicas que são aplicadas para um único usuário e outras que necessitam de um grupo de 
usuários para serem executadas. Como resultado, as seguintes técnicas foram escolhidas: Brainstorming, Entrevistas, JAD, Questionários e Prototipação Descartável.

A técnica utilizada para auxiliar o Analista na seleção da técnica de elicitação foi a Matriz de Decisão. Esta técnica consiste em ponderar informações (parâmetros retirados do trabalho de [Belgamo e Martins, 2000] e [Batista e Carvalho, 2003]) que levarão a possíveis soluções de interesses (Técnicas de Elicitação) em linhas e colunas de uma matriz. Para cada intercessão de linha e coluna é aplicada uma pontuação (pesos atribuídos para cada técnica em relação a cada parâmetro) e normalmente, a análise do resultado na matriz de decisão é feita considerando que o maior valor ponderado constituirá, possivelmente, a melhor decisão a ser tomada.

Ao preencher a Matriz de Decisão, o Analista de Requisitos deve informar se ele deseja avaliar técnicas que podem ser aplicadas para coletar requisitos com apenas um usuário ou com um grupo de usuários. Esta é uma restrição para que seja efetuado o cálculo da pontuação total. Em seguida, são definidos quais parâmetros devem ser priorizados na escolha da técnica que será utilizada para o levantamento de requisitos de acordo com características do projeto (levantadas nas atividades anteriores). Esta definição é realizada através da atribuição do grau de relevância (nota) para cada parâmetro. Este peso pode variar de 0 a 5 pontos, indicando o grau de relevância que o parâmetro tem dentro do projeto. Ao final, todas as técnicas que atendem à restrição da fonte de opção de requisitos terão suas respectivas pontuações totais calculadas.

Os resultados da matriz são gerados através do somatório das pontuações intermediárias obtidas para cada técnica. Estas pontuações são calculadas através da multiplicação do peso pela nota, atribuída pelo Analista, para cada técnica em relação a cada parâmetro. A técnica mais adequada será a que obtiver a maior pontuação total.

A quarta atividade, "Aplicar a técnica para elicitar os requisitos" consiste em coletar e listar os requisitos para desenvolvimento do sistema através da técnica, escolhida na atividade anterior, pelo Analista com o suporte deste processo.

$\mathrm{Na}$ quinta e ultima atividade, "Elaborar a lista de requisitos", o Analista de Requisitos elabora a Lista de Requisitos, baseado nos requisitos identificados durante a elicitação. A Lista de Requisitos deve conter uma descrição do sistema, a descrição dos requisitos e o critério de aceitação, que consiste nas condições necessárias para verificar se o requisito foi implementado. Esta lista é inspecionada por um processo de verificação intermediaria, para que as atividades posteriores à elicitação de requisitos possam ser realizadas.

\subsubsection{Experimentos e Resultados Obtidos com a Primeira Versão do Processo}

O objetivo da primeira versão do processo de elicitação foi auxiliar o Analista de Requisitos na escolha da técnica de elicitação a ser utilizada e prover uma maior colaboração entre os envolvidos no processo. Experimentos foram realizados e seus resultados analisados conforme os passos a seguir.

A proposta para validação desta primeira versão do processo consistiu em aplicá-lo em um projeto piloto e posteriormente em estudos de caso de projetos reais. $\mathrm{O}$ objetivo do projeto piloto foi verificar se a descrição das atividades poderia ser facilmente seguida e se os artefatos gerados eram suficientes e necessários para o processo de elicitação. 
Uma planilha para contabilização do tempo e custo despendidos e uma Checklist de Verificação para validação da Lista de Requisitos foram elaboradas para registrar os dados referentes aos estudos de caso. Estes documentos não fazem parte do processo e foram utilizados apenas com o propósito de realizar a validação dos experimentos.

Nas próximas seções, é descrito em maior detalhe como a verificação foi realizada, os estudos de caso e os resultados alcançados. O objetivo dos estudos de caso apresentados foi comparar uma elicitação realizada com o processo proposto, em sua primeira versão, com outra realizada com uma metodologia distinta de forma a identificar qual proporcionou melhor atendimento aos objetivos do Cliente.

\subsubsection{Descrição do mecanismo para Verificação}

Os experimentos foram realizados em 4 projetos em 3 empresas diferentes. Para cada projeto havia 2 analistas com perfis e experiências similares; um realizava a elicitação utilizando o a primeira versão do processo proposto neste trabalho e outro utilizava um procedimento próprio da empresa.

Para validar a primeira versão do processo, em [Barbosa et al., 2009], dados referentes a tempo e custo das elicitações foram coletados. Além disso, o cliente verificou os requisitos levantados em ambas as elicitações (com e sem o processo proposto) por meio da Checklist de Verificação. Esta checklist contempla itens de verificação que medem o grau de completeza, precisão, consistência e clareza de cada requisito identificado e também da Lista de Requisitos como um todo. Desta forma, foi possível atestar o grau de conformidade dos requisitos elicitados em relação ao Contexto das informações e a Qualidade dos requisitos.

Para medir se os parâmetros priorizados pelo Analista, antes da elicitação, foram mais bem atendidos com o processo proposto, nesta primeira versão, cada item da Checklist de Verificação foi relacionado a um parâmetro analisado. Esse relacionamento indica que o item de verificação, se atendido correta e completamente, leva ao atendimento do parâmetro correspondente. A classificação dos itens da Checklist foi baseada na definição de [Belgamo e Martins, 2000] [Batista e Carvalho, 2003].

A Checklist de Verificação é uma planilha na qual os requisitos identificados são dispostos em linhas enquanto os itens de verificação são dispostos nas colunas. Na intersecção de uma linha e uma coluna, o cliente deve informar se o item em questão está conforme, parcialmente conforme, não conforme ou se ele não sabe avaliar. Para medir de forma simples a verificação dos requisitos, a cada uma das possíveis respostas foram atribuídos valores. Itens conformes receberam valor igual a 1; parcialmente conformes receberam valor igual a 0,5 e itens não conformes valor igual a 0 .

Para melhor comparar os resultados dentro de cada estudo de caso, foi definido o Universo de Requisitos, que corresponde ao número total de requisitos únicos identificados em ambas elicitações (com e sem o processo), ou seja, todos os requisitos do sistema identificados pelo Cliente. Esse número é definido de acordo com a verificação realizada pelo Cliente.

\subsubsection{Consolidação dos Resultados}

A Tabela 1 apresenta os resultados obtidos nos estudos de caso realizados em [Barbosa et al., 2009] para validar a primeira versão do processo proposto de forma sumarizada. 
Os resultados confirmam que os requisitos elicitados com o uso desta primeira versão do processo proposto apresentaram maior Qualidade e permitiram identificar melhor o Contexto do projeto na concepção do cliente. Embora em alguns estudos, a mesma técnica tenha sido utilizada em ambas execuções (com e sem o processo), na avaliação do cliente, o resultado da elicitação realizada com o processo proposto apresentou maior qualidade e uma melhor compreensão do contexto da aplicação.

Tabela 1. Resultados Sumarizados dos Estudos de Caso (primeira versão do processo de elicitação)

\begin{tabular}{|c|c|c|c|c|c|c|c|c|}
\hline $\begin{array}{l}\text { Dados Obtidos/ } \\
\text { Itens Avaliados }\end{array}$ & \multicolumn{2}{|c|}{$\begin{array}{l}\text { Estudo de Caso } 1 \\
\text { Sistema de Viagens }\end{array}$} & \multicolumn{2}{|c|}{$\begin{array}{c}\text { Estudo de Caso } 2 \\
\text { Comércio Eletrônico }\end{array}$} & \multicolumn{2}{|c|}{$\begin{array}{c}\text { Estudo de Caso } 3 \\
\text { Bug Tracking }\end{array}$} & \multicolumn{2}{|c|}{$\begin{array}{c}\text { Estudo de Caso } 4 \\
\text { Comércio em Bairro }\end{array}$} \\
\hline \multirow{2}{*}{$\begin{array}{l}\text { Universo de } \\
\text { Requisitos }\end{array}$} & \multicolumn{2}{|c|}{21} & \multicolumn{2}{|c|}{23} & \multicolumn{2}{|c|}{24} & \multicolumn{2}{|c|}{35} \\
\hline & $\begin{array}{c}\text { Com } \\
\text { Processo }\end{array}$ & $\begin{array}{c}\text { Sem } \\
\text { Processo }\end{array}$ & $\begin{array}{c}\text { Com } \\
\text { Processo }\end{array}$ & $\begin{array}{c}\text { Sem } \\
\text { Processo }\end{array}$ & $\begin{array}{c}\text { Com } \\
\text { Processo }\end{array}$ & $\begin{array}{c}\text { Sem } \\
\text { Processo }\end{array}$ & $\begin{array}{c}\text { Com } \\
\text { Processo }\end{array}$ & $\begin{array}{c}\text { Sem } \\
\text { Processo }\end{array}$ \\
\hline Técnica & Protótipo & Entrevista & Entrevista & Entrevista & Entrevista & Entrevista & Brainstorming & Entrevista \\
\hline Tempo (hrs) & 6 & 8 & 2,5 & 2 & 2,5 & 1 & 3,5 & 1 \\
\hline $\begin{array}{l}\mathbf{N}^{0} \text { Requisitos } \\
\text { Identificados }\end{array}$ & 21 & 6 & 23 & 15 & 24 & 11 & 35 & 11 \\
\hline $\begin{array}{l}\text { Porcentagem } \\
\text { (Universo)* }\end{array}$ & $100 \%$ & $29 \%$ & $100 \%$ & $65 \%$ & $100 \%$ & $46 \%$ & $100 \%$ & $31 \%$ \\
\hline Qualidade*** & $98 \%$ & $100 \%$ & $100 \%$ & $74 \%$ & $98 \%$ & $75 \%$ & $95 \%$ & $51 \%$ \\
\hline $\begin{array}{c}\text { Qualidade } \\
\text { Ponderada**** }\end{array}$ & $98 \%$ & $29 \%$ & $100 \%$ & $48 \%$ & $98 \%$ & $34 \%$ & $95 \%$ & $16 \%$ \\
\hline Contexto***** & $100 \%$ & $50 \%$ & $100 \%$ & $50 \%$ & $100 \%$ & $25 \%$ & $100 \%$ & $25 \%$ \\
\hline$*$ & \multicolumn{8}{|c|}{$\begin{array}{l}\text { Representa a porcentagem do número de requisitos levantados, em cada elicitação (com e sem o processo), em relação } \\
\text { ao número de requisitos total identificados pelo cliente (Universo de Requisitos). Indica o quão completa está a lista } \\
\text { de requisitos em relação ao Universo de Requisitos, para cada estudo de caso. }\end{array}$} \\
\hline$* *$ & \multicolumn{8}{|c|}{$\begin{array}{l}\text { Representa o grau de conformidade dos requisitos, de acordo com avaliação do cliente, em cada elicitação } \\
\text { isoladamente (com e sem o processo), em relação aos itens da Checklist de Verificação relacionados à Qualidade. }\end{array}$} \\
\hline$* * *$ & \multicolumn{8}{|c|}{$\begin{array}{l}\text { Representa o grau de conformidade dos requisitos identificados em cada elicitação, de acordo com a avaliação do } \\
\text { cliente, em relação aos itens da Checklist de Verificação relacionados à Qualidade ponderados pelo Universo de } \\
\text { Requisitos. Este indicador permite a comparação dos resultados das elicitações (com e sem o processo) em cada } \\
\text { estudo de caso, relacionados à Qualidade dos requisitos identificados, dentro de um universo único de requisitos. }\end{array}$} \\
\hline$* * * *$ & \multicolumn{8}{|c|}{$\begin{array}{l}\text { Representa o grau de conformidade dos requisitos identificados, de acordo com avaliação do cliente, em cada } \\
\text { elicitação em relação aos itens da Checklist de Verificação relacionados ao Contexto. Indica o quão dentro do } \\
\text { contexto do domínio de negócio os requisitos identificados nos estudos de caso (com e sem o processo) estão. }\end{array}$} \\
\hline
\end{tabular}

Também é possível observar na Tabela 1 que, em alguns casos, a elicitação realizada com o processo proposto demandou um tempo maior para ser realizada se comparada a elicitação sem o processo, contudo, essa diferença é vista como investimento para um melhor levantamento dos requisitos. Nestes estudos de caso, em que a utilização do processo apresentou um maior tempo de execução, o parâmetro "Tempo gasto para a elicitação" não era uma restrição e os indicadores apresentaram maior qualidade para os requisitos elicitados durante o levantamento realizado com a primeira versão do processo proposto neste trabalho.

\subsubsection{Validando a etapa de Seleção da Técnica de Elicitação proposta na primeira versão do Processo de Elicitação}

Durante os experimentos realizados com a primeira versão proposta para o processo de elicitação, através da aplicação das técnicas indicadas pelo processo e os resultados 
obtidos, foi observado que pode haver uma diferenciação ainda maior entre as técnicas em relação aos parâmetros pelos quais as mesmas foram classificadas. Nos trabalhos utilizados como base, elaborados por [Belgamo e Martins, 2000] [Batista e Carvalho, 2003], há parâmetros com o mesmo peso para várias técnicas. As diferenças entre as técnicas podem ser mais bem exploradas diferenciando seus pesos ou mesmo incluindo novos parâmetros.

Com o objetivo de verificar se a primeira versão do processo proposto indicou a técnica de elicitação de forma correta, os resultados obtidos foram confrontados, em [Barbosa e Barbosa, 2009], com os resultados dos trabalhos publicados recentemente por [Dieste, Lopez e Ramos, 2008] e [Carrizo, Dieste e Juristo, 2008]. Estes trabalhos apresentam classificações mais detalhadas sobre as técnicas de elicitação que poderiam ser usadas. Estas classificações foram conduzidas através de uma profunda revisão da literatura sobre tais técnicas.

Em [Carrizo, Dieste e Juristo, 2008] foi elaborado um framework que auxilia na identificação da técnica mais adequada para um determinado cenário de projeto. Para alcançar este resultado, foram analisadas na literatura informações sobre quando e onde o uso de cada técnica era mais adequado e posteriormente um conjunto de parâmetros, relacionados à eficácia e eficiência de cada técnica que poderiam influenciar na elicitação de acordo com o projeto, foram determinados. Para concluir, todas as informações foram compiladas em um framework que combina técnicas de elicitação com parâmetros relacionados a projetos.

No framework elaborado, cada técnica recebeu o seu nível de adequação para cada parâmetro relacionado a projetos de software, de acordo com as possíveis classificações que este parâmetro poderia receber. Os níveis de adequação propostos, para uma possível classificação, foram: Adequado, Indiferente e Não Adequado.

A Matriz de Decisão usada nos experimentos realizados com a primeira versão do processo proposto neste trabalho foi atualizada por [Barbosa e Barbosa, 2009] para considerar os mesmos critérios e valores definidos em [Carrizo, Dieste e Juristo, 2008].

Os mesmos analistas que conduziram os experimentos iniciais, descritos neste trabalho utilizando o processo proposto, preencheram a Matriz de Decisão atualizada considerando as características e/ou restrições dos mesmos projetos que elicitaram anteriormente. Desta forma foi possível realizar uma comparação entre a técnica indicada no experimento realizado com a matriz de decisão da primeira versão do processo proposto e a indicada pela matriz de decisão atualizada.

O objetivo da comparação foi analisar se a matriz de decisão atualizada apontaria diferentes técnicas para os experimentos realizados anteriormente, e se estas poderiam ser consideradas mais adequadas para cada cenário.

Surpreendentemente, a técnica Questionário foi indicada como a técnica a ser utilizada para o levantamento de requisitos em todos os estudos de caso realizados, descritos em [Barbosa e Barbosa, 2009], nesta execução da Matriz de Decisão, mesmo para projetos em que havia um grupo de usuários disponível para realizar a elicitação.

Embora Questionário seja uma técnica utilizada quando se identifica necessidade de coletar informações de muitos usuários ao mesmo tempo [Lauesen, 2002], considera-se que os fornecedores de requisitos respondem ao questionário 
individualmente, sendo esta uma técnica aplicada para um indivíduo e não para um grupo.

Nestes novos experimentos, os requisitos não foram coletados utilizando a técnica de Questionário para verificar se esta técnica poderia proporcionar um melhor resultado se aplicada conforme propõe o processo de elicitação de requisitos com foco na seleção da técnica de elicitação.

Foi possível verificar, com base no resultado destes últimos experimentos, e analisando o framework proposto por [Carrizo, Dieste e Juristo, 2008] que Questionário foi uma técnica classificada como "Adequada" para a maioria, em torno de 86,36\%, dos parâmetros relacionados a projetos de software avaliados. Esta análise indica que na maioria dos casos, provavelmente, nenhuma outra técnica poderia ser selecionada se considerarmos apenas a classificação proposta pelos autores.

Ao analisar os resultados obtidos nos experimentos realizados por [Barbosa et al., 2009] e [Barbosa e Barbosa, 2009], verificamos que os parâmetros propostos por [Belgamo e Martins, 2000] e [Batista e Carvalho, 2003] permitiam considerar no momento da escolha da técnica de elicitação apenas aspectos relacionados ao domínio/contexto do projeto, como por exemplo, prazo e custo. Já os parâmetros propostos por [Carrizo, Dieste e Juristo, 2008] permitiam considerar aspectos relacionados ao perfil do elicitante e do informante além do domínio do problema.

Diante desta análise concluímos que para oferecer um maior apoio na seleção da técnica de elicitação é importante considerar, na tomada de decisão, características do(s) usuário(s), analista(s) de requisitos, escopo e do domínio/contexto do projeto. Esta conclusão foi baseada também no referencial teórico que enfatiza tanto a importância de existir uma colaboração entre elicitantes e informantes durante a definição e gerenciamento dos requisitos quanto a necessidade de estabelecer critérios relacionados ao escopo do projeto para escolher uma técnica de elicitação.

Evoluímos o processo de elicitação de requisitos com foco na seleção da técnica para sua segunda versão para que fosse possível agregar ao mesmo as melhorias diagnosticadas nas experiências anteriores. Tais melhorias são o objetivo deste trabalho e são apresentadas na próxima seção.

\subsection{Evolução do Processo de Elicitação de Requisitos Proposto (Segunda Versão)}

A segunda versão do processo com foco na seleção da técnica de elicitação permanece com cinco atividades, conforme a primeira versão, porém apresenta algumas atualizações, conforme ilustrado na Figura 1 e descrito a seguir. 


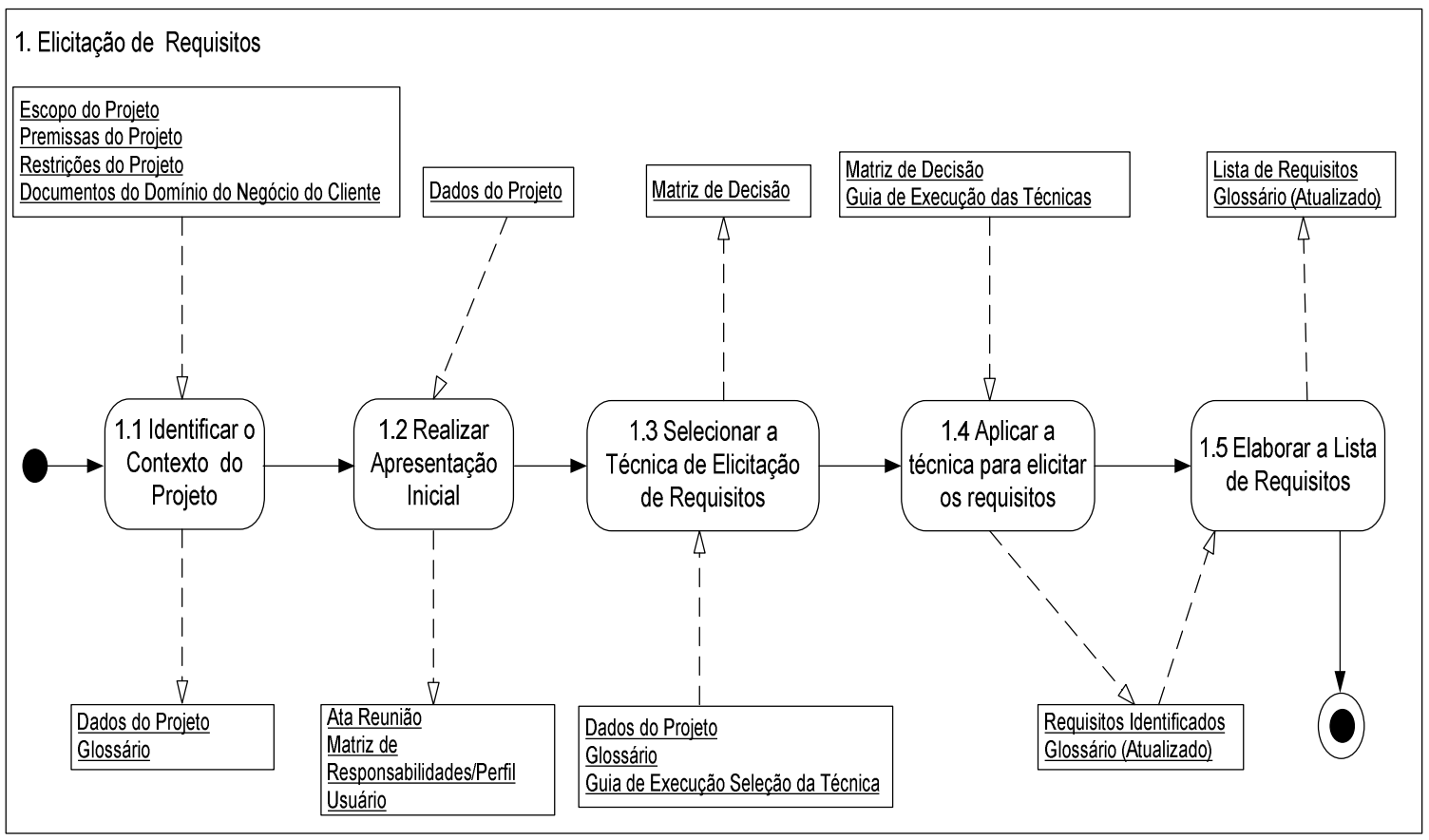

Figura 1. Segunda versão do Processo de Elicitação de Requisitos proposto (Atualizado)

\subsubsection{Atividade: Identificar o Contexto do Projeto}

Esta atividade não sofreu alteração em relação à atividade "Identificar o Contexto do Projeto “ proposta em [Barbosa et al., 2009], descrita na seção 4.1 deste trabalho.

\subsubsection{Atividade: Realizar Apresentação Inicial}

Esta atividade tem como propósito realizar uma reunião para conscientizar os envolvidos sobre a importância da colaboração do(s) usuário(s) dentro do processo de elicitação de requisitos, uma vez que esta colaboração estará associada ao sucesso do resultado final do sistema que será desenvolvido.

Nesta versão atualizada, além de coletar informações sobre os papéis e responsabilidades dos usuários envolvidos, o Analista de Requisitos também coleta informações sobre o perfil dos envolvidos no processo de elicitação, em relação à experiência dos mesmos em elicitação de requisitos e sobre o domínio da aplicação que será desenvolvida. Estas informações são estruturadas em uma Matriz de Responsabilidades/Perfil do Usuário. Esta Matriz permite que o Analista de Requisitos reúna informações e identifique o perfil de cada um dos usuários envolvidos, facilitando assim a condução da elicitação e a identificação de parâmetros que influenciam a seleção da técnica. Uma Ata de Reunião é gerada para documentar os temas abordados e repassá-los a todos os envolvidos. 


\subsubsection{Atividade: Selecionar a Técnica de Elicitação de Requisitos}

Esta atividade foca em orientar o Analista de Requisitos na escolha da técnica a ser utilizada com base em parâmetros relacionados ao perfil do Analista e Usuário(s) envolvidos e ao projeto.

\subsubsection{Elaboração da Matriz de Decisão para apoio à tomada de decisão}

Para que fosse possível considerar um universo significativo de parâmetros, no momento da seleção da técnica, foram selecionados para compor esta nova versão do processo alguns relacionados ao perfil do(s) usuário(s) e analista(s) de requisitos, a partir do trabalho elaborado por [Carrizo, Dieste e Juristo, 2008]. Foram selecionados também outros relacionados a características do domínio/contexto e escopo do projeto, com base nos trabalhos de [Belgamo e Martins, 2000] e [Batista e Carvalho, 2003].

Um exemplo de parâmetro escolhido é a "Fonte de obtenção dos requisitos". Este foi considerado uma restrição para a seleção, pois há técnicas que focam na elicitação voltada para grupo de usuários e outras aplicadas individualmente. Os parâmetros selecionados e suas definições segundo os autores são apresentados, na Tabela 2:

\section{Tabela 2. Parâmetros que devem ser considerados no momento da escolha da técnica de elicitação}

\begin{tabular}{|c|c|c|c|}
\hline Par: & relacionados ao Elicit & $\begin{array}{l}\text { e (Analista de Requisitos), Informante (Usuário) e Do } \\
\text { [Carrizo, Dieste e Juristo, 2008] }\end{array}$ & ínio do Problema \\
\hline \multirow{13}{*}{ 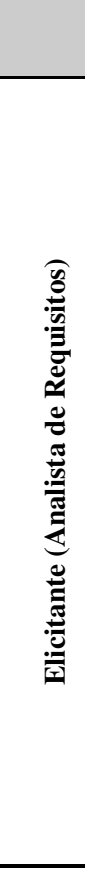 } & Parâmetro & Descrição do Parâmetro & $\begin{array}{c}\text { Possíveis } \\
\text { Classificações }\end{array}$ \\
\hline & \multirow{3}{*}{$\begin{array}{l}\text { Treinamento em } \\
\text { técnicas de elicitação }\end{array}$} & \multirow{3}{*}{$\begin{array}{l}\text { Indica o nível de conhecimento e prática, que o Analista } \\
\text { de Requisitos possui, sobre a aplicação de técnicas } \\
\text { (independente da técnica) para realizar levantamento de } \\
\text { requisitos }\end{array}$} & Alto \\
\hline & & & Baixo \\
\hline & & & Zero (Nenhum) \\
\hline & \multirow{3}{*}{$\begin{array}{l}\text { Experiência } \quad \text { com } \\
\text { elicitação }\end{array}$} & \multirow{3}{*}{$\begin{array}{l}\text { Indica o nível experiência do Analista de Requisitos em } \\
\text { levantamento de requisitos baseado no número de projetos } \\
\text { em que ele participou como elicitante }\end{array}$} & Alto \\
\hline & & & Médio \\
\hline & & & Baixo \\
\hline & \multirow{3}{*}{$\begin{array}{l}\text { Experiência com } \\
\text { técnicas de elicitação }\end{array}$} & \multirow{3}{*}{$\begin{array}{l}\text { Indica a quantidade de vezes que o Analista de Requisitos } \\
\text { levantou requisitos utilizando alguma técnica de elicitação }\end{array}$} & Alto \\
\hline & & & Baixo \\
\hline & & & Zero (Nenhuma) \\
\hline & \multirow{3}{*}{$\begin{array}{l}\text { Familiaridade com o } \\
\text { domínio }\end{array}$} & \multirow{3}{*}{$\begin{array}{l}\text { Indica o nível de conhecimento que o Analista de } \\
\text { Requisitos possui sobre o domínio de negócio da } \\
\text { aplicação que será desenvolvida }\end{array}$} & Alto \\
\hline & & & Baixo \\
\hline & & & Zero (Nenhum) \\
\hline \multirow{4}{*}{ 莺 } & \multirow{2}{*}{$\begin{array}{l}\text { Consenso entre os } \\
\text { informantes }\end{array}$} & \multirow{2}{*}{$\begin{array}{l}\text { Indica o nível de consenso entre as informações, iniciais, } \\
\text { que o(s) usuário(s) possuem em relação ao problema e/ou } \\
\text { domínio de negócio. }\end{array}$} & Alto \\
\hline & & & Baixo \\
\hline & \multirow[t]{2}{*}{ Interesse do informante } & \multirow{2}{*}{$\begin{array}{l}\text { Indica o nível de interesse do(s) usuário(s) em } \\
\text { participar(em) da(s) sessões de elicitação }\end{array}$} & Alto \\
\hline & & & Baixo \\
\hline
\end{tabular}




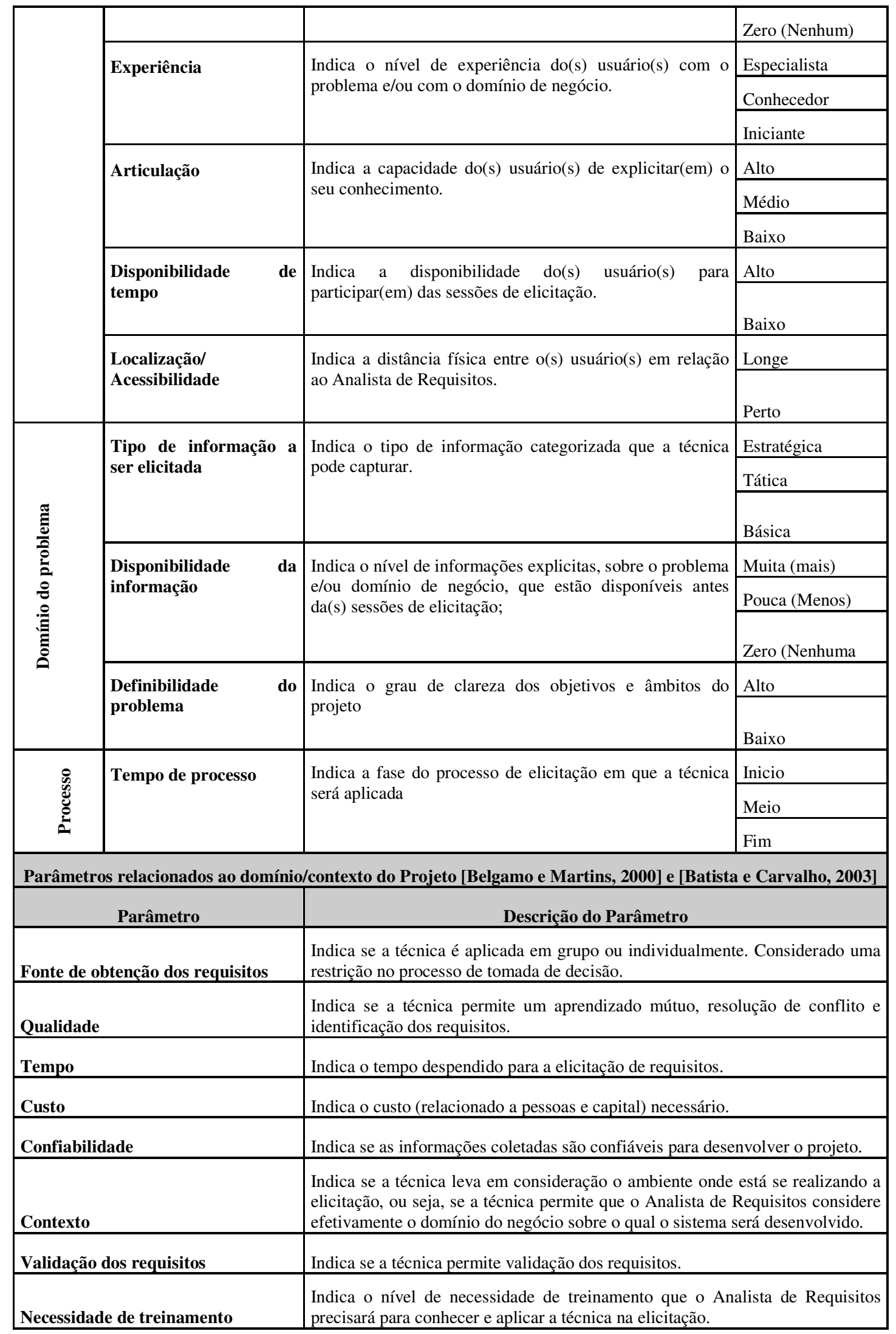


Com o conjunto de técnicas e parâmetros definidos, deve então ser aplicada a tomada de decisão para escolha da técnica de elicitação.

Com o objetivo de estruturar melhor este processo de seleção da técnica, a Matriz de Decisão proposta, nesta segunda versão do processo, é composta por duas matrizes, a primeira foi denominada "Matriz de Decisão - Perfil dos Envolvidos (Analista e Usuário)", e a segunda "Matriz de Decisão - Critérios Específicos do Projeto". Desta forma, a seleção da técnica mais indicada passa por duas etapas e abrange características relacionadas ao perfil dos envolvidos e do projeto em questão, avaliando as mesmas técnicas em ambos os casos para se obter o resultado final. Esta foi a principal mudança realizada no processo em relação a sua primeira versão.

A Matriz de Decisão - Perfil dos Envolvidos (Analista e Usuário), que deve ser utilizada na primeira etapa da atividade de seleção da técnica, foi construída estruturando seus dados em linhas e colunas:

- Linhas: Representam cada um dos parâmetros relacionados ao perfil do analista de requisitos, usuário e domínio do problema que são levados em consideração no momento da escolha da técnica;

- Colunas: Representam as técnicas de elicitação (é importante ressaltar que este é o mesmo conjunto de técnicas utilizadas na "Matriz de Decisão - Critérios Específicos do Projeto").

Para cada técnica de elicitação, foram atribuídas pontuações em relação aos parâmetros relacionados ao perfil do Analista e do Usuário, indicando o nível de adequação que aquela técnica se encontra em relação à classificação fornecida para o parâmetro em questão.

Para melhor exemplificar, considere o parâmetro "Experiência com elicitação". Caso o Analista tenha participado de:

- 05 ou mais elicitações, a experiência e classificação para o parâmetro é "Alta";

- 02 a 05 elicitações, sua experiência e classificação para o parâmetro é "Média";

- Menos de 02 elicitações, sua experiência e classificação para o parâmetro é "Baixa".

Caso a classificação atribuída para o parâmetro "Experiência com elicitação" tenha sido, por exemplo, Alta, as técnicas serão classificadas nos níveis de adequação "Adequado", "Indiferente" ou "Não Adequado", de acordo com o nível proposto pelos autores [Carrizo, Dieste e Juristo, 2008]. Para construir a Matriz de Decisão estes níveis foram mapeados para valores numéricos para calcular o resultado parcial desta etapa.

Na Figura 2, é possível demonstrar, por exemplo que, para as técnicas, Prototipação e Brainstorming, o fato do Analista de Requisitos ter participado de poucos levantamentos de requisitos (independente do uso ou não de técnicas) é irrelevante para que as mesmas possam ser utilizadas. Contudo neste caso em que o Analista apresenta baixa experiência não é adequado que se faça a utilização da técnica JAD [Carrizo, Dieste e Juristo, 2008]. 


\begin{tabular}{|c|c|c|c|c|c|c|c|c|}
\hline & & Matr & $z$ de Decisão & - Perfil dos E & Envolvidos (Ana & ista e Usuário) & & \\
\hline & & $\begin{array}{l}\text { Colunas de } \mathrm{Pr} \\
\text { Analista } \mathrm{d}\end{array}$ & $\begin{array}{l}\text { eenchimento do } \\
\text { Requisitos }\end{array}$ & Entrevista & Questionário & Prototipação & JAD & Brainstorming \\
\hline & $\begin{array}{l}\text { Restrição: Selecione a Fonte } \\
\text { de obtenção dos requisitos }\end{array}$ & & upo & Individuo & Individuo & Individual/Grupo & Grupo & Grupo \\
\hline & & Classificą̧ão & $\begin{array}{l}\text { Justificativa da } \\
\text { Classificaçãa }\end{array}$ & Pontuação & Pontuação & Pontuação & Pontuaçäo & Pontuação \\
\hline & $\begin{array}{l}\text { Treinamento em técnicas de } \\
\text { elicitaçäo }\end{array}$ & Baixo & & 0 & 0 & 0 & 0 & 2 \\
\hline \pm & Experiência com elicitação & Baixo & . & 0 & 0 & 0 & -2 & 0 \\
\hline 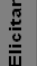 & $\begin{array}{l}\text { Experiência com técnicas de } \\
\text { elicitação }\end{array}$ & Baixo & & 0 & 0 & 2 & 2 & 2 \\
\hline & Familiaridade com o dominio & Zero & & 0 & 0 & -2 & -2 & 2 \\
\hline & $\begin{array}{l}\text { Consenso entre os } \\
\text { informantes }\end{array}$ & Alto & & 0 & 0 & 2 & 0 & 0 \\
\hline है & Interesse do informante & Alto & & 0 & 0 & 2 & 2 & 2 \\
\hline$\stackrel{\Xi}{E}$ & Experiência & Conhecedor & & 0 & 0 & 2 & 2 & 2 \\
\hline (2) & Articulaçāo & Alto & & 0 & 0 & 2 & 2 & 2 \\
\hline & Disponibilidade de tempo & Baixo & & 0 & 0 & 0 & -2 & -2 \\
\hline & Localizaçãol Acessibilidade & Perto & & 0 & 0 & 2 & 2 & 2 \\
\hline ㅇำ & $\begin{array}{l}\text { Tipo de informação a ser } \\
\text { elicitada }\end{array}$ & Tática & & 0 & 0 & 2 & 2 & 2 \\
\hline 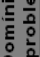 & $\begin{array}{l}\begin{array}{l}\text { Disponibilidade da } \\
\text { informaçăo }\end{array} \\
\end{array}$ & Maior & & 0 & 0 & 2 & 2 & 0 \\
\hline 0 & Definibilidade do problema & Alto & & 0 & 0 & 2 & -2 & 2 \\
\hline$\frac{0}{2}$ & Tempo de processo & Início & & 0 & 0 & 0 & 2 & 2 \\
\hline & & RESL & LTADO PARCIAL 1 & 0 & 0 & 16 & 8 & 18 \\
\hline
\end{tabular}

Figura 2. Matriz de Decisão - Perfil dos Envolvidos (Analista e Usuários)

Para cada parâmetro existe um conjunto de possíveis classificações prédefinidas. Para que se possam obter as pontuações de cada técnica e o resultado parcial desta etapa, o Analista informa a classificação de cada parâmetro da matriz, de acordo com o perfil do(s) Usuário, do Analista de Requisitos e do domínio do problema do projeto em questão (levantados nas atividades anteriores). O Analista deve registrar uma justificativa para cada classificação atribuída para análises futuras.

Os resultados parciais da Matriz de Decisão Perfil dos Envolvidos (Analista e Usuário), ou seja, da primeira etapa da atividade de seleção da técnica, são gerados, para cada técnica, através do somatório das suas respectivas pontuações obtidas nesta etapa.

A Matriz de Decisão - Critérios Específicos do Projeto, que deve ser utilizada na segunda etapa da atividade de seleção da técnica, é construída estruturando seus dados em linhas e colunas:

- Linhas: Representam cada um dos parâmetros relacionados a projetos de software que são levados em consideração no momento da escolha da técnica;

- Colunas: Representam as técnicas de elicitação. (é importante ressaltar que este é o mesmo conjunto de técnicas utilizadas na "Matriz de Decisão - Perfil dos Envolvidos (Analista e Usuário)").

Nesta segunda matriz, para cada técnica de elicitação, foi atribuído um peso em relação aos parâmetros relacionados ao domínio/contexto e escopo de projetos, indicando o grau que aquela técnica atende ao parâmetro em questão. Os pesos são valores que correspondem às classificações propostas pelos autores [Belgamo e Martins, 
2000] e [Batista e Carvalho, 2003]. Nestes, cada técnica recebeu classificações de "Baixo" a "Alto" para cada parâmetro avaliado. Neste trabalho, as classificações foram mapeadas para valores de 1 a 3 para calcular o resultado parcial da Matriz correspondente. Como o objetivo é atribuir a maior pontuação à técnica mais adequada, os valores foram assinalados aos parâmetros dependendo do objetivo desejado minimizar ou maximizar o efeito do parâmetro. Assim, parâmetros que devem ser maximizados, como Qualidade, receberam pesos de 1 a 3, para classificações de Baixo a Alto. Parâmetros que devem ser minimizados, como Tempo, receberam pesos de 1 a 3 , representando a escala de Alto a Baixo.

Na Figura 3, é possível demonstrar, por exemplo, que as técnicas Brainstorming, JAD e Prototipação Descartável propõem uma metodologia que visa coletar requisitos com alta qualidade. A técnica Questionário, embora apresente um tempo de execução médio em relação à JAD não permite que o contexto do projeto, como informações sobre o domínio de negócio, possa ser explorado no momento da elicitação [Belgamo e Martins, 2000] e [Batista e Carvalho, 2003].

O Analista define quais parâmetros devem ser priorizados na escolha da técnica que será utilizada para elicitação dos requisitos de acordo com características do projeto (levantadas nas atividades anteriores). Esta definição é realizada através da atribuição do grau de relevância (nota) para cada um dos parâmetros. Este peso pode variar de 0 a 2 pontos, onde 0 indica irrelevância, 1 indica uma baixa relevância e 2 indica uma alta relevância do parâmetro para o projeto. O Analista deve registrar uma justificativa para cada nota para análises futuras.

Os resultados parciais da Matriz de Decisão - Critérios Específicos do Projeto são gerados através do somatório das pontuações intermediárias obtidas para cada técnica. Estas pontuações são calculadas através da multiplicação do peso pela nota, atribuída pelo Analista, para cada técnica em relação a cada parâmetro.

\begin{tabular}{|c|c|c|c|c|c|c|c|c|c|c|c|c|}
\hline & & & atriz de & Decisão & - Critéri & os Espec & cíficos c & do Projet & & & & \\
\hline Parâmetros & $\begin{array}{l}\text { Colunas } \\
\text { Anali }\end{array}$ & $\begin{array}{l}\text { de Preenchimento do } \\
\text { lista de Requisitos }\end{array}$ & Entre & evista & Quest & onário & $\begin{array}{l}\text { Protot } \\
\text { Desc }\end{array}$ & $\begin{array}{l}\text { tipação } \\
\text { artável }\end{array}$ & $\mathrm{J} /$ & D & Brainst & torming \\
\hline $\begin{array}{l}\text { Restriçäo: } \\
\text { Selecione a } \\
\text { Fonte de }\end{array}$ & & Grupo & Indi & viduo & Indi & iduo & Grupo I & Individuo & Grt & po & Gru & upo \\
\hline & Sua Nota & Justificativa da Nota & Peso & Pontuação & Peso & Pontuação & Peso & Pontuação & Peso & Pontuação & Peso & Pontuação \\
\hline Qualidade & 2 & & 2 & 0 & 1 & 0 & 3 & 6 & 3 & 6 & 3 & 6 \\
\hline Tempo Gasto & 2 & & 2 & 0 & 2 & 0 & 2 & 4 & 1 & 2 & 2 & 4 \\
\hline Custo & 2 & & 2 & 0 & 2 & 0 & 1 & 2 & 1 & 2 & 2 & 4 \\
\hline Confiabilidade & 2 & & 3 & 0 & 2 & 0 & 3 & 6 & 3 & 6 & 2 & 4 \\
\hline Contexto & 1 & & 3 & 0 & 1 & 0 & 3 & 3 & 3 & 3 & 3 & 3 \\
\hline $\begin{array}{l}\overline{\text { Validar }} \\
\text { requisitos }\end{array}$ & 1 & & 3 & 0 & 0 & 0 & 3 & 3 & 3 & 3 & 0 & 0 \\
\hline $\begin{array}{l}\text { Esforço } \\
\text { despendido } \\
\text { com } \\
\text { treinamento }\end{array}$ & 2 & & 2 & 0 & 1 & 0 & 1 & 2 & 1 & 2 & 2 & 4 \\
\hline & & RESULTADO PARCIAL 2 & $\begin{array}{l}\text { Pontuação } \\
\text { Parcial }\end{array}$ & 0 & $\begin{array}{c}\text { Pontuação } \\
\text { Parcial }\end{array}$ & 0 & \begin{tabular}{|c} 
Pontuação \\
Parcial
\end{tabular} & 26 & $\begin{array}{c}\text { Pontuação } \\
\text { Parcial }\end{array}$ & 24 & \begin{tabular}{|c|} 
Pontuação \\
Parcial
\end{tabular} & 25 \\
\hline
\end{tabular}

Figura 3. Matriz de Decisão - Critérios Específicos do Projeto 
Para se obter a técnica mais indicada para o levantamento de requisitos do software, calcula-se a Pontuação Final para cada técnica que compõe a Matriz de Decisão. Esta pontuação é calculada, para cada técnica, a partir da soma entre a Pontuação Parcial obtida na primeira etapa da seleção e a Pontuação Parcial obtida na segunda etapa da seleção. A técnica mais adequada será a que obtiver a maior pontuação total. A Figura 4 ilustra o procedimento descrito acima.

\begin{tabular}{|l|c|c|c|c|c|}
\hline \multicolumn{1}{|c|}{ Técnica } & Entrevista & Questionário & $\begin{array}{c}\text { Prototipação } \\
\text { Descartável }\end{array}$ & JAD & Brainstorming \\
\hline MD - Caract. Analist e Usuarios & 0 & 0 & 16 & 8 & 18 \\
\hline MD - Criterios Esp Projeto & 0 & 0 & 26 & 24 & 25 \\
\hline PONTUAÇÁO FINAL & 0 & 0 & 42 & 32 & 43 \\
\hline
\end{tabular}

Figura 4. Cálculo Final para Seleção da Técnica

A seção seguinte descreve como o Analista de Requisitos deve proceder para executar a atividade: "Selecionar a Técnica de Elicitação de Requisitos" durante a etapa de elicitação de requisitos seguindo a nova versão do processo proposto.

\subsubsection{Descrição do Processo de Seleção da Técnica}

Antes de levantar os requisitos do sistema, o Analista de Requisitos deverá selecionar uma técnica que o auxilie na elicitação. Considerando que alguns parâmetros relacionados ao perfil do Analista, do Usuário e as características projeto influenciam a escolha da técnica que deverá ser utilizada, o Analista de Requisitos deverá utilizar a Matriz de Decisão, proposta, na segunda versão, do processo de elicitação, para auxiliar a tomada de decisão.

Para guiar o Analista de Requisitos, foi elaborado um Guia de Execução para Seleção da Técnica. Este guia contempla um conjunto de definições, procedimentos e orientações que auxiliam o Analista nesta etapa.

Em um primeiro momento, baseado nas informações coletadas nas atividades posteriores relacionadas ao perfil do Analista e do(s) Usuário(s) que atuarão no projeto e as características do(s) mesmo(s), o Analista preenche a "Matriz de Decisão - Perfil dos Envolvidos" informando se a técnica deve ser aplicada para coletar requisitos com um único individuo ou com um grupo de usuários, ou seja, a fonte de obtenção de requisitos. Posteriormente, ele informa as classificações de cada parâmetro listado na matriz para o projeto em questão. Por exemplo, para o parâmetro "Familiaridade com o domínio", o Analista deverá indicar o nível de conhecimento que o mesmo possui sobre o domínio de negócio da aplicação em intervalo que varia de Zero (Nenhum), Baixo ou Alto. Ao final desta primeira etapa, todas as técnicas que atendem a restrição sobre a fonte de obtenção de requisitos terão suas respectivas pontuações parciais calculadas.

Em um segundo momento, baseado nas informações coletadas nas atividades anteriores relacionadas ao domínio e escopo do projeto, o Analista preenche a Matriz de Decisão - Critérios Específicos do Projeto. O Analista informa uma nota para cada um dos parâmetros do processo, refletindo assim a importância ou a existência de restrições em relação a este parâmetro no projeto. Por exemplo, para o parâmetro "Qualidade", o Analista deverá se perguntar: "Em que grau o projeto demanda uma técnica que enfoque na qualidade dos requisitos elicitados?". O Analista deverá atribuir uma nota de 0 (Nenhum) a 2 (alto). Para todos os parâmetros listados, o Analista deve informar uma 
nota. Ao final desta segunda etapa, todas as técnicas que atendem à restrição sobre a fonte de obtenção de requisitos terão suas respectivas pontuações parciais calculadas.

A pontuação final, para cada uma das técnicas que atende a restrição da fonte de obtenção de requisitos, é calculada através da soma das pontuações parciais obtidas por cada técnica em cada uma das etapas da seleção.

A técnica que obtiver a maior pontuação final será a indicada para que o Analista de Requisitos aplique-a no levantamento de requisitos do software. Entretanto, a atividade de seleção atua como uma forma de orientação na escolha da técnica. $O$ Analista de Requisitos poderá escolher uma técnica diferente da que obteve a maior pontuação final, devido à proximidade dos resultados ou por outro motivo justificável. A escolha do Analista deverá ser aprovada pelo Gerente de Projeto.

O Analista de Requisitos deverá registrar a técnica indicada pelo processo, a técnica que ele optou por utilizar, a justificativa da escolha e a técnica que realmente será utilizada com aprovação do Gerente de Projeto para a elicitação. As informações geradas durante a execução do processo deverão ser armazenadas para cada projeto. Estas podem compor uma base histórica auxiliando projetos futuros.

É importante ressaltar que a classificação e o grau de relevância de cada parâmetro podem variar de acordo com o projeto. Assim, o mesmo Analista poderá atribuir uma classificação na primeira etapa da seleção, ou um grau de relevância, na segunda etapa da seleção, distintos para o mesmo parâmetro em projetos diferentes.

\subsubsection{Atividade: Aplicar a Técnica para elicitar os requisitos}

Esta etapa não sofreu alteração em relação a atividade "Aplicar a Técnica para elicitar os requisitos " proposta em [Barbosa et al., 2009], descrita na seção 4.1 deste trabalho.

\subsubsection{Atividade: Elaborar a Lista de Requisitos}

Esta etapa não sofreu alteração em relação a atividade "Elaborar a Lista de Requisitos“" proposta em [Barbosa et al., 2009], descrita na seção 4.1 deste trabalho.

\subsection{Estudos de Caso e Resultados obtidos para Validação da Segunda versão do Processo de Elicitação Proposto}

Ao executar os experimentos da primeira versão do Processo de Elicitação de Requisitos em empresas de desenvolvimento de software, verificamos que a utilização do mesmo contribui de forma significativa para melhorar a qualidade dos requisitos elicitados e proporcionou melhor atendimento aos objetivos do Cliente.

Para esta segunda versão do processo, não ocorreu uma comparação entre o processo atualizado e metodologias distintas, uma vez que esta avaliação já foi realizada anteriormente. A proposta para validação desta nova versão do processo consistiu em aplicá-lo em estudos de caso de projetos reais e avaliar o resultado obtido quanto à qualidade e ao atendimento da expectativa do cliente quanto aos requisitos elicitados.

Novamente foi utilizada a Checklist de Verificação, já detalhada na seção 4.1.1.1 e os resultados foram analisados baseados no Universo de Requisitos (número total de requisitos únicos identificados) indicado pelo Cliente. 
A Tabela 3 apresenta os resultados obtidos nos estudos de caso em uma empresa de desenvolvimento para a segunda versão do processo proposto de forma sumarizada.

Tabela 3. Resultados Sumarizados dos Estudos de Caso (segunda versão do processo de elicitação)

\begin{tabular}{l|c|c}
\multicolumn{1}{c|}{$\begin{array}{c}\text { Projetos de Software } \\
\text { Dados Obtidos/ Itens Avaliados }\end{array}$} & Meeting & Gestor de Metas \\
\hline Universo de Requisitos & 30 & 27 \\
Técnica & Brainstorming & JAD \\
Tempo (hrs) & 9 & 14,5 \\
$\mathbf{N}^{\mathbf{0}}$ Requisitos Identificados & 30 & 27 \\
Porcentagem (Universo)* & $100 \%$ & $100 \%$ \\
Qualidade* & $100 \%$ & $100 \%$ \\
Qualidade Ponderada* & $100 \%$ & $100 \%$ \\
Contexto* & $100 \%$ & $100 \%$ \\
\hline \multicolumn{2}{c}{$*$} & Conceitos explicados na Tabela 1 \\
\hline
\end{tabular}

\section{Conclusões e Trabalhos Futuros}

O objetivo deste trabalho foi evoluir o processo de elicitação de requisitos com foco na seleção da técnica de elicitação, de modo a considerar uma quantidade significativa de parâmetros no momento da escolha da técnica e prover uma maior colaboração entre os envolvidos no processo.

Para a primeira versão do Processo de Elicitação de Requisitos com foco na Seleção da Técnica de Elicitação foram realizados experimentos com o objetivo de avaliar o processo proposto em relação a outras metodologias utilizadas em empresas de desenvolvimento de software para a elicitação de requisitos.

De maneira geral, os requisitos elicitados seguindo a primeira versão do processo apresentaram resultados melhores em relação às elicitações realizadas com outras metodologias. Isso se deve aos fatores-chave priorizados pelo processo; o primeiro relacionado aos critérios para selecionar a técnica de elicitação mais adequada para o levantamento dos requisitos e o segundo por permitir maior colaboração e interatividade entre Analistas, Gerentes e Clientes.

Contudo, foi observado que poderia haver uma diferenciação ainda maior entre as técnicas em relação aos parâmetros pelos quais as mesmas foram classificadas. As diferenças entre as técnicas poderiam ser exploradas diferenciando seus pesos ou mesmo novos parâmetros poderiam ser incluídos. Ao realizar uma nova pesquisa na literatura foram encontrados trabalhos recentes [Dieste, Lopez e Ramos, 2008] [Carrizo, Dieste e Juristo, 2008] que apresentavam classificações mais detalhadas sobre as técnicas de elicitação que poderiam ser usadas neste sentido.

A proposta desta segunda versão consistiu em evoluir o processo aumentando a quantidade de parâmetros a serem considerados no momento da escolha da técnica, mais adequada, de tal forma que fosse possível considerar não apenas aqueles

\footnotetext{
${ }^{1}$ http://www.besconsulting.com.br/besEnterpriseSolutions.aspx
} 
relacionados ao escopo do projeto, como na primeira versão, mas também aqueles relacionados a características do Analista e do(s) Usuário(s) que participam da elicitação além de manter a proposta de colaboração entre os envolvidos.

Os novos experimentos mostraram que as elicitações realizadas, com a segunda versão do processo, contemplaram requisitos que correspondem às necessidades do cliente, de acordo com as verificações realizadas pelo mesmo. Os novos resultados apresentaram requisitos dentro do contexto e da qualidade esperada pelo cliente.

Pretende-se ainda realizar experimentos para acompanhar a maturidade dos requisitos até o fim do desenvolvimento após aplicar o processo. Assim, poder-se-á observar se os requisitos priorizarão os atributos identificados pelo Cliente.

\section{Referências}

Barbosa, G. A. R.; Barbosa, M. W.; Assis. H; Fernandes. U; Silva. I (2009) "Um processo de elicitação de requisitos com foco na seleção da técnica de elicitação". In: Simpósio Brasileiro de Qualidade de Software (SBQS) Ouro Preto - Minas Gerais.

Barbosa, M. W.; Barbosa, G. A. R. (2009) "Towards the selection of the most suitable elicitation technique through a defined requirements elicitation process". In: Software Engineering and Knowledge Engineering (SEKE`09) Boston - USA.

Batista, E. A; Carvalho, A. M. B. R. (2003) "Uma Taxonomia Facetada para Técnicas de Elicitação de Requisitos". WER03 - Workshop em Engenharia de Requisitos.

Belgamo, A.; Martins, L. E. G (2000). "Estudo Comparativo sobre as Técnicas de Elicitação de Requisitos do Software". In: XX Congresso Brasileiro da Sociedade Brasileira de Computação (SBC), Curitiba - Paraná.

Carrizo, D.; Dieste, O. e Juristo, N. (2008) "Study of Elicitation Techniques Adequacy". In Workshop on Engenharia de Requisitos (WER 2008), Barcelona, Espanha.

Dieste, O.; Lopez, M. e Ramos, F. (2008) "Updating Systematic Review about Selection of Software Requirements Elicitation Techniques". In Workshop on Engenharia de Requisitos (WER 2008), Barcelona, Espanha.

Freitas, D. P.; Borges, M. R. S; Araújo, R. M. (2007) "Colaboração e Negociação na Elicitação de Requisitos" In: X Workshop Iberoamericano de Ingeniería de Requisitos y Ambientes de Software (IDEAS 07), Isla de Margarita

Hickey, A. M. e Davis, A. M. (2002) "Requirements Elicitation and Elicitation Technique Selection: A Model for Two Knowledge-Intensive Software Development Processes". In: Proceedings of Hawaii International Conference on System Sciences

Lauesen, S. (2002) "Software Requirements Styles and Techniques". Elicitation. England: A Personal Education Limited, 2002. Cap.8 p.331-372.

Pfleeger, S. L. (2004) "Engenharia de software: teoria e prática". Identificando Requisitos 2a .ed. São Paulo: Prentice Hall. Cap.4 p.111-138.

Robbins, S. P (2005). "Comportamento Organizacional. Percepção e Tomada de Decisões Individual”. 11 a . Ed. São Paulo: Pearson Prentice Hall. Cap.5, p.103 -125.

Standish Group International (1995). "The Chaos Report"; http://net.educause.edu/ir/library/pdf/NCP08083B.pdf. 